\title{
Reversible Visible Watermarking for H.264/AVC Encoded Video
}

\author{
Reuben A. Farrugia \\ Department of Communications and Computer Engineering \\ University of Malta, Msida \\ MSD 2080, Malta \\ Email: reuben.farrugia@um.edu.mt
}

\begin{abstract}
Visible watermarked images and videos are generally used to convey ownership information. However, the visible watermark is generally irreversible and thus authenticated users cannot recover the original image or video quality after watermark extraction. This poses a limitation in various scenarios including military, law and medical applications.

This paper presents a novel reversible visible watermarking scheme for H.264/AVC encoded video sequences. The proposed approach reversibly embeds the residual information that will then be used by the decoder to recover the original image. The residual information is losslessly compressed using the ZLib Deflector algorithm to minimize the information to be embedded. The compressed information is then encrypted using the 128-bit Advanced Encryption Standard (AES). Simulation results clearly demonstrate the superiority of the proposed scheme to current state of the art where Peak Signal-to-Noise Ration (PSNR) gains of up to $7 \mathrm{~dB}$ were achieved.
\end{abstract}

\section{INTRODUCTION}

The recent growth of computer networks and multimedia systems has contributed to the proliferation of multimedia content. However, the availability of multimedia editing software has raised the issue of unauthorized manipulation of proprietary material [1]. Visible watermarking techniques have been extensively used to protect copyrighted material. However, traditional approaches, such as [2], [3], are not able to recover the original image/video quality after watermarking extraction.

The methods proposed in [4], [5] apply removable visible watermarking schemes where authenticated users are allowed to approximate the visible watermark. Nonetheless, these methods only manage to recover an approximate version of the original image after watermark extraction and are therefore unsuitable for military, law and medical applications [6].

There are several reversible visible watermarking schemes that can be employed for such applications [7], [8], [9], [10]. However, these methods are susceptible to quantization errors provided by standard image/video compression standards. Therefore, these methods are not suitable for most Internet applications where multimedia content needs to be compressed prior transmission. In previous work, the same author has presented a reversible watermarking scheme for JPEG image compression [11]. However, this method cannot be directly integrated within current video compression standards, mainly due to the spatio-temporal prediction mechanisms being employed by video standards.
This paper presents an adaptation of the reversible visible watermarking scheme presented in [11] for H.264/AVC video coding. The proposed method computes the residual error caused by the embedded watermark which is losslessly compressed using the ZLib Deflector algorithm [12] and then encrypted using the Advanced Encryption Standard (AES) [13]. The resulting information is embedded within the transform coefficients of every macroblock (MB) pair using the Reversible Contrast Mapping (RCM) algorithm published in [14]. The simulation results clearly show that the proposed mechanism outperforms the state of the art approach where Peak Signal-to-Noise Ratio (PSNR) gains of up to $7 \mathrm{~dB}$ were registered.

The structure of this paper is as follows. Section II provides a detailed description of each component involved in the Reversible Visible Watermarking Embedding process while the Reversible Visible Watermark Extraction process is described in section III. Section IV presents the testing environment and presents the simulation results. The final comments and conclusion are delivered in section $\mathrm{V}$.

\section{REVERSIBLE VISIBLE WATERMARK EMBEDDING}

A high level description of the proposed Reversible Visible Watermark Embedding process for video content is illustrated in Fig. 1. The original frame $I$ is first fed to the Visible Watermarking Embedding process which inserts a visible watermark within its Region of Interest (ROI) to generate the frame $I_{W}$. Given that the embedded watermark directly affects the image content, it makes this process irreversible.

The resulting watermarked frame $I_{W}$ is then compressed using the Video Encoder 1 process which employs motion estimation and spatial prediction of the standard H.264/AVC encoder [15] to minimize the residual error $E_{W C}$ to be entropy coded. The motion vectors and modes selected for each Macroblock (MB) are registered in the Control Information module. This information is then used by the Video Encoder 2 to compress the original image $I$ which outputs the residual error $E_{C}$. This process computes neither motion estimation nor mode decision, but relies solely on the information contained within the Control Information module, thus significantly reducing the complexity of the proposed system.

The difference between the residual errors of the compressed watermarked image $E_{W C}$ and the compressed image 


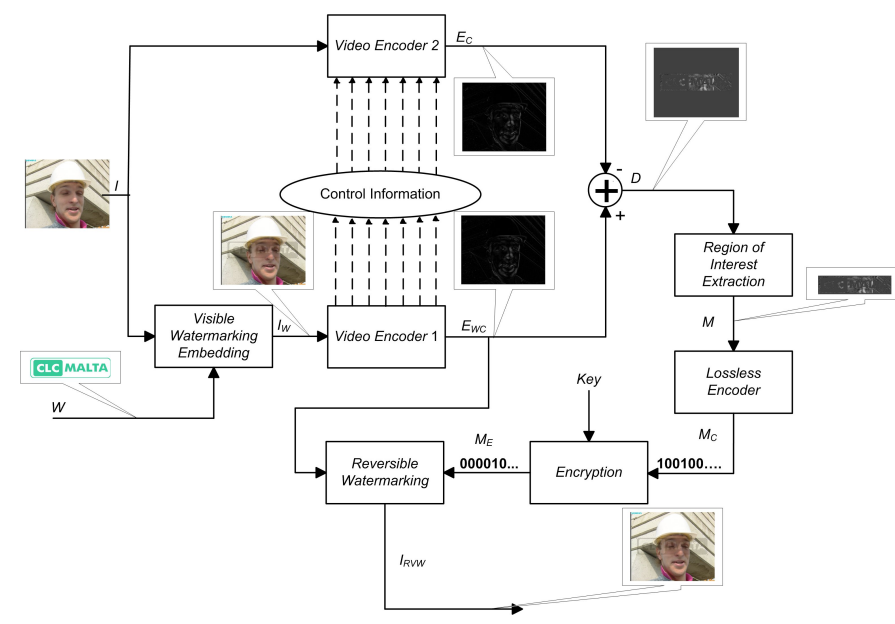

Fig. 1. Schematic diagram of the Reversible Visible Watermark Embedding process.

$E_{C}$ provides the discrepancy image $D$. This image contains 0 values outside the ROI, while having low magnitude values within the ROI. Therefore, in order to minimize the information to be embedded within the image, only the message $M$ encompassing the Region of Interest within $D$ is losslessly encoded and encrypted. The encrypted message $M_{E}$ is then embedded within the watermarked image $I_{W}$ using a reversible watermarking approach. More information about each individual module is provided in the following sub-sections.

\section{A. Visible Watermarking Embedding}

The Visible Watermarking Embedding process is used to perceptibly insert a watermark $W$ into a primary image $I$ so that the watermark is visible by the human eye. The purpose of these watermarks is to be noticeable without significantly reducing the quality of the image. The method adopted in this work considers the human vision system (HVS model) and the image content to insert the watermark without significantly degrading the perceptual quality of the image/video content. This process dissects the host image $I$ into non-overlapping $8 \times 8$ blocks. The watermark pattern $W$ is then adaptively embedded into the host image using

$$
I_{n}^{W}(i, j)= \begin{cases}\left\lfloor\alpha_{n} I_{n}(i, j)\right\rfloor, & \text { if } W(i, j)=1 \\ I_{n}(i, j), & \text { Otherwise }\end{cases}
$$

where $\lfloor\bullet\rfloor$ represents the floor function, $I_{n}^{W}$ and $I_{n}$ denote the $n^{\text {th }} 8 \times 8$ block of the watermarked image $I_{W}$ and the host image $I$ respectively, $i$ and $j$ are spatial coordinates within the block and $\alpha_{n}$ is the $n^{\text {th }}$ adaptive scaling coefficient. The scaling coefficients $\alpha_{n}$ are dependent on both the human perception and image content and determine the visibility of the watermark pattern. More information about the derivation of these scaling coefficients can be found in [7] and [11].

\section{B. Video Encoder}

The Video Encoder process employs the standard H.264/AVC encoder to compress the supplied video content.
This process employs spatial prediction and motion estimation to minimize the residual error to be encoded. The encoder derives the residual information to be encoded by subtracting the predicted frame from the original frame. The resulting residual information is de-correlated using the discrete cosine transform (DCT) transformation and quantized to keep the least amount of information while still achieving an acceptable level of image quality. The quantized transform coefficients are then inverse quantized and inverse transformed to recover the residual error $E$ which also includes the quantization error introduced by the lossy nature of the standard video codec.

The proposed method employs two Video Encoder processes. The Video Encoder 1 process receives the watermarked frame $I_{W}$ and computes motion estimation and spatial prediction to compress the video. The resulting modes selected and motion vectors are then stored in the Control Information module while the resulting residual error $E_{W C}$ is outputted. On the other hand, the Video Encoder 2 process receives the original video stream $I$ and compresses it using the motion vectors and mode selected available in the Control Information module. This is done to ensure synchronization between the two processes and to minimize the computational complexity of the proposed system.

\section{Lossless Encoder and Encryption}

The discrepancy message $D$ is derived by subtracting the residual $E_{C}$ from the residual $E_{W C}$. As it can be seen from Fig. 1, the non-zero coefficients reside only within the Region of Interest. Therefore, in order to minimize the data to be embedded, the Region of Interest is extracted from the discrepancy message $D$ to generate the message $M$ and this information is then passed through the Lossless Encoder process.

The Lossless Encoder process adopts a simple encoding strategy, where each pixel in $M$ is represented by a 10-bit codeword. The Most Significant Bit (MSB) is used to flag whether the pixel is a watermark (1) or not (0). The second MSB represents the polarity of the coefficient where negative coefficients are marked by a 1 . The remaining 8 -bits represent the magnitude of the coefficients of the residual error message $M$. Given that the decoder needs some extra information to be able to decode the encoded message (e.g. watermark dimensions and coordinates of the ROI), this information is concatenated to $M$ prior to lossless encoding. The resulting 10-bit codewords are then compressed using the public domain lossless ZLib Deflector algorithm [12]. Furthermore, in order to enhance the security, a 128-bit Advanced Encryption Standard (AES) [13] is used to encrypt the information to be embedded so that only authenticated users can recover the original image.

\section{Reversible Watermarking}

The Reversible Watermarking process adopts the Reversible Contrast Mapping (RCM) mechanism presented in [14] to embed the encrypted information $M_{E}$ within the watermarked image $I_{W}$. The RCM algorithm was used since it is reported to 
provide high-capacity data embedding without the requirement of the transmission of any additional side information.

The RCM algorithm considers $x$ and $y$ to be a pair of coefficients whose values reside in the range $[0, L]$. The forward RCM algorithm transforms the pixel pairs according to

$$
\dot{x}=2 x-y, \quad \underline{y}=2 y-x
$$

In order to prevent overflow and underflow, the transformed pixel pairs are restricted within the range

$$
0 \leq \dot{x} \leq L, 0 \leq \dot{y} \leq L
$$

The original coefficients can be recovered using the following inverse transformation

$$
x=\left\lceil\frac{2}{3} \dot{x}+\frac{1}{3} y^{\prime}\right\rceil, \quad y=\left\lceil\frac{1}{3} \dot{x}+\frac{2}{3} y^{\prime}\right\rceil
$$

where $\lceil\bullet\rceil$ is the ceil function.

The RCM method substitutes the Least Significant Bit (LSB) of $\dot{x}$ and $y$. The LSB of $\dot{x}$ is set to 1 to indicate a transformed pair while 0 otherwise. The information bit $b$ is then embedded within the LSB of $y$. More information about this method can be found in [14].

The method proposed in [7], which is considered as the state of the art method, applies the RCM algorithm in the spatial domain. However, as shown in [11], the RCM method cannot be applied in the spatial domain when considering compressed images since the embedded information will be distorted by the Quantization process of the image/video codec. It was further shown that the RCM method can modify the transformed coefficients prior entropy coding, thus making recovery of the original transform coefficients possible. However, the transform coefficients provide different levels of distortions [16], and therefore it is more desirable to embed the information within high frequency coefficients which provide the least distortion to the human vision system.

Therefore, considering a $P \times Q$ image, the Reversible Watermarking scans the blocks column-wise and grabs the first two neighboring $4 \times 4$ transformed blocks. It embeds the first bit within the coordinate $(3,3)$ which corresponds to the highest frequency coefficient. The remaining $\frac{P \times Q}{32}-1$ bits are then stored within the coefficient with coordinates $(3,3)$ of the remaining neighbor blocks. Once all the blocks have been used this process goes back to the first two neighboring blocks and embeds the second $\frac{P \times Q}{32}$ bits within the coefficient coordinate $(3,2)$. This process proceeds until either the whole bitstream is embedded or else when all the transform coefficients are used. It is important to notice that the RCM algorithm can be used to embed information more than once within the same coefficients. However, this will contribute to major distortions within the image. Therefore, the transform coefficients were only used once for embedding. The Reversible Watermarking process then outputs the image $I_{R V C}$ which is then entropy encoded and transmitted.

\section{REVERSIBLE VISIBLE WATERMARK EXTRACTION}

The Reversible Visible Watermark Extraction process inverts the computations performed by the Reversible Visible Watermark Embedding process. It receives the entropy coded information and decodes it to recover the image $I_{R V W}$. The Reversible Data Extraction process then computes the inverse RCM function to extract the encrypted message $M_{E}$ and the visible watermarked residual $E_{W}$. The message $M_{E}$ is then decrypted and decoded to derive the message $M$.

The message $M$ contains the residual watermark within the Region of Interest together with additional information suitable to reconstruct the discrepancy image $D$. The images $D$ and $E_{W C}$ are then summed to generate $E_{C}$. The $V$ ideoDecoder then is able to recover the original compressed video sequence $I_{C}$.

\section{Simulation Results}

The proposed Reversible Visible Watermarking Scheme was implemented using the $\mathrm{C}++$ programming language. The raw video sequences considered in these simulation results were encoded using a Common Intermediate Format (CIF) resolution at 30fps with a format IPPP ... using the Baseline Profile of H.264/AVC. The video was encoded using only the $4 \times 4$ transform size. The Quantization Parameter is set to a default of 20 unless otherwise specified. The logos employed in these experiments included some of the logos available in [17].

Table I illustrates the performance of the proposed $R e$ versible Visible Watermarking Embedding mechanism. It can be clearly seen that the compression efficiency provided by the Lossless Encoder process is between 1.8 and 2.5. This compression efficiency is almost constant and thus not affected by the logo size. It can be further noticed that the larger the size of the logo to be embedded the lower is the quality of the Watermarked Video $I_{W}$. This is quite intuitive since larger logos need to modify more DCT coefficients thus inevitably reducing the perceptual quality of the Watermarked Video.

TABLE I

Performance Analysis of the Proposed Reversible Visible WATERMARKING EMBEDDING PROCEsS

\begin{tabular}{|l|l|l|l|l|l|}
\hline Logo & Width & Height & $\begin{array}{l}M_{E} \text { Size } \\
\text { (Bytes) }\end{array}$ & $\begin{array}{l}\text { Comp. } \\
\text { Ratio }\end{array}$ & $\begin{array}{l}I_{W} \text { PSNR } \\
(\mathbf{d B})\end{array}$ \\
\hline ATLSS & 163 & 216 & 14408 & 2.4436 & 26.2887 \\
DARPA & 130 & 263 & 16461 & 2.0770 & 26.9543 \\
Censcir & 82 & 225 & 8747 & 2.1093 & 30.1921 \\
Robotics & 130 & 108 & 5623 & 2.4969 & 31.4413 \\
CIT & 82 & 225 & 8472 & 2.1778 & 31.7764 \\
HCII & 65 & 65 & 2253 & 1.8753 & 37.4408 \\
\hline
\end{tabular}

The proposed system was compared to the Yang method [7] which was adapted for compressed video. As it can be seen from Fig. 2, the quality of the proposed method is superior to the method adopted in [7]. In fact, it can be seen that no matter how much information is reversible embedded the proposed system manages to recover the original quality for authenticated users. This is mainly due to the fact that the information is being embedded on the compressed transform 
coefficients. On the other hand, the performance of the state of the art approach [7] degrades with increasing embedding information since the information is hidden within the spatial domain which is corrupted by the Quantization process.

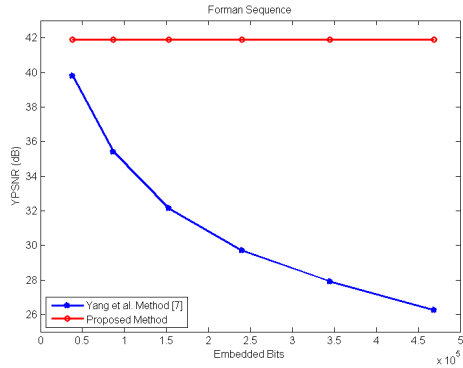

Fig. 2. Performance of the Yang method [7] and the proposed method at different number of embedded bits
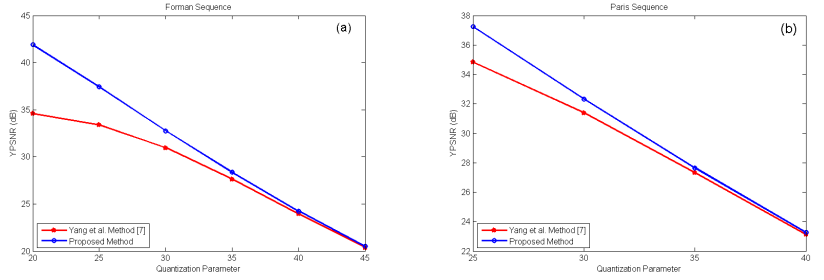

Fig. 3. Performance of the Yang [7] and the proposed method at different Quantization Parameters (a) Foreman (b) Paris Sequence

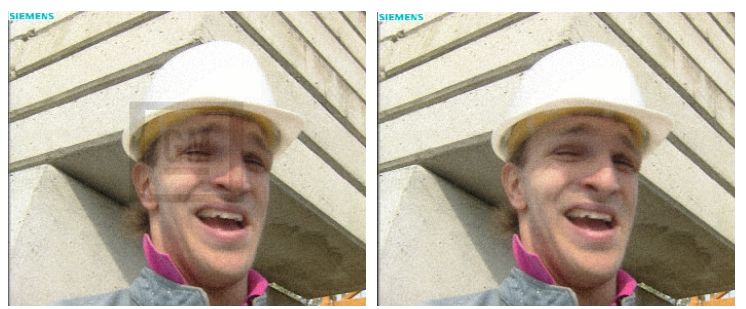

Fig. 4. Subjective results when using the (a) method proposed by Yang [7] and the (b) Proposed method. QP was set to 30.

The performance of the proposed scheme was further analyzed using different Quantization Parameters. As shown in Fig. 3, the proposed system clearly outperforms the method presented in [7] where PSNR gains of up to $7 \mathrm{~dB}$ were achieved. The superiority of the proposed method is more evident in Fig. 4 where it can be seen that the method proposed by Yang does not manage to extract the reversible information since it was corrupted by quantization errors induced by the lossy Video Encoder process. The quantization errors generally provide syntax and semantic violations in the De-Encryption and Lossless Decoding processes which are thus not able to recover the original embedded information. On the other hand the proposed method manages to recover the original compressed video when the user is authenticated.

\section{COMments ANd CONCLUSion}

This paper has presented a novel reversible visible watermarking scheme for H.264/AVC encoded video. The proposed method computes the information required by the decoder to recover the original compressed video when receiving the watermarked video sequence. The additional information is reversibly embedded within the transform coefficients of the watermarked video. The authenticated users are then enabled to extract the information hidden within the transform coefficients to recover the original compressed image.

The experimental results have shown the superiority of the proposed system where PSNR gains of up to $7 \mathrm{~dB}$ was registered relative to the state of the art approach. It was further shown that the information to be hidden can be compressed with compression efficiency between 1.8 and 2.5. Further research is required in order to reduce the distortion provided by the RCM method. Furthermore, watermark estimation functions can be employed in order to reduce the energy within the message $D$ to be decoded.

\section{REFERENCES}

[1] W. Min and L. Bede, "Watermarking for image authentication," Image Processing, International Conference on, vol. 2, p. 437, 1998.

[2] B.-B. Huang and S.-X. Tang, "A contrast-sensitive visible watermarking scheme," IEEE MultiMedia, vol. 13, no. 2, pp. 60-66, 2006.

[3] S. P. Mohanty, K. R. Ramakrishnan, and M. S. Kankanhalli, "A DCT domain visible watermarking technique for images," in Multimedia and Expo, 2000. ICME 2000. 2000 IEEE International Conference on, New York, NY, USA, pp. 1029-1032.

[4] Y. Hu, S. Kwong, and J. Huang, "An algorithm for removable visible watermarking," Circuits and Systems for Video Technology, IEEE Transactions on, vol. 16, no. 1, pp. 129 - 133, jan. 2006.

[5] Y. Yang, X. Sun, H. Yang, and C.-T. Li, "Removable visible image watermarking algorithm in the discrete cosine transform domain," Journal of Electronic Imaging, vol. 17, no. 3, p. 033008, 2008. [Online]. Available: http://link.aip.org/link/?JEI/17/033008/1

[6] A. Alattar, "Reversible watermark using the difference expansion of a generalized integer transform," Image Processing, IEEE Transactions on, vol. 13, no. 8, pp. $1147-1156$, aug. 2004.

[7] Y. Yang, X. Sun, H. Yang, C.-T. Li, and R. Xiao, "A contrast-sensitive reversible visible image watermarking technique," Circuits and Systems for Video Technology, IEEE Transactions on, vol. 19, no. 5, pp. 656 -667 , may 2009.

[8] Y. Hu and B. Jeon, "Reversible visible watermarking and lossless recovery of original images," Circuits and Systems for Video Technology, IEEE Transactions on, vol. 16, no. 11, pp. $1423-1429$, nov. 2006.

[9] S.-K. Yip, O. Au, C.-W. Ho, and H.-M. Wong, "Lossless visible watermarking," in Multimedia and Expo, 2006 IEEE International Conference on, 9-12 2006, pp. $853-856$.

[10] H.-M. Tsai and L.-W. Chang, "A high secure reversible visible watermarking scheme," in Multimedia and Expo, 2007 IEEE International Conference on, 2-5 2007, pp. $2106-2109$.

[11] R. A. Farrugia, "A reversible visible watermarking scheme for compressed images," in MELECON 2010 - 2010 15th IEEE Mediterranean Electrotechnical Conference, 26-28 2010, pp. 212 -217.

[12] (2010) The zlib website. [Online]. Available: http://www.zlib.net/

[13] Advamced Encryption Standard (AES), FIPS PUB Std. 197, 2001.

[14] D. Coltuc and J.-M. Chassery, "Very fast watermarking by reversible contrast mapping," Signal Processing Letters, IEEE, vol. 14, no. 4, pp. $255-258$, april 2007.

[15] T. Wiegand, G. Sullivan, G. Bjontegaard, and A. Luthra, "Overview of the h.264/avc video coding standard," Circuits and Systems for Video Technology, IEEE Transactions on, vol. 13, no. 7, pp. $560-576$, july 2003.

[16] A. Watson, "Visually optimal det quantization matrices for individual images," in Data Compression Conference, 1993. DCC '93., 1993, pp. $178-187$.

[17] (2010) Institute of complex engineering systems (ices) website. [Online]. Available: http://www.ices.cmu.edu/logos.html 